Research Article

\title{
Analytical Investigation of Transient Wave Propagation in One-Dimensional Unsaturated Poroelastic Materials
}

\author{
Hao Xiong (iD \\ School of Civil Engineering and Architecture, Taizhou University, Taizhou 318000, China \\ Correspondence should be addressed to Hao Xiong; haosyong@126.com
}

Received 15 September 2020; Revised 28 October 2020; Accepted 31 October 2020; Published 17 November 2020

Academic Editor: Junyan Yi

Copyright $\odot 2020$ Hao Xiong. This is an open access article distributed under the Creative Commons Attribution License, which permits unrestricted use, distribution, and reproduction in any medium, provided the original work is properly cited.

\begin{abstract}
As a dynamic response, the wave propagation phenomenon usually varies with different media. In this study, the dynamic response of unsaturated poroelastic materials to an impulse load has been analytically investigated. The governing equations, in the Laplace domain, of the unsaturated poroelastic soil in terms of the variables $u_{s}$ (solid displacement), $p_{f}$ (pore fluid pressure), and $p_{a}$ (pore air pressure) will be derived. These equations will be simplified in a one-dimensional form. The solutions that include the dynamic response to the vertical displacement of solid particles and to the variations of fluid and air pressures will be provided which are applicable for an arbitrary loading form. The solutions were validated with the results for saturated soil presented in the literature. The effect of material parameters on dynamic response was analysed through a series of parameter studies. It was found that increasing the porosity or fluid saturation effectively increases the amplitude of fluid and air pressures as well as the wave velocity. Although increasing the fluid saturation leads to solid displacement gradually decreases gradually, it results in increasing the amplitude of fluid and air pressure. The fluid saturation increasing above 0.9 results in wave travels faster significantly. The variation of fluid intrinsic permeability has little influence on the soil dynamic response until it reaches a high level. The findings of this study can help for better understanding of one-dimensional wave propagation in unsaturated soil.
\end{abstract}

\section{Introduction}

The soil in the civil engineering field is known as a mixture of solid particles, fluids, and gases, and it is commonly treated as an ideal elastic and fluid-filled porous medium, namely, saturated poroelastic material $[1,2]$.

In the past few years, the load-induced saturated soil response has been widely investigated by analytical method. For example, Philippacopoulos [3] calculated the explicit general solutions of displacements and stresses of a homogeneous half-space model in the wavenumber-frequency domain via Helmholtz decompositions and Fourier transforms. Yazdi et al. [4] analysed the dynamic response of a horizontal layer model to a disturbance induced by a strip footing. They found an exact solution in the frequency domain and proposed a finite element scheme for complex geometries. Jin and Liu [5] investigated the motion of a three-dimensional poroelastic half-space produced by a horizontal buried loading using integral transform techniques. In addition to the analytical method, numerical methods have also been extensively used in load-induced saturated soil response problems. For instance, Cheng et al. [6] presented a singular integral equation technique, called the boundary element method, for solving dynamic poroelasticity problems. Moreover, other methods can be exemplified by the stiffness matrix method proposed by Mesgouez and Lefeuve-Mesgouez [7] and Wang and Rokhlin [8]; transmission and reflection matrix method by Pei et al. [9], and generalized transfer matrix method by Liu and Zhao [10].

Regarding the governing equations, in practice, the soil is considered as a partially saturated multiphase material consisting of a solid, a pore fluid (water or oil), and the air. The governing equations of a linear elastic porous medium permeated by two immiscible Newtonian fluids were established by Tuncay and Corapcioglu [11]. Based on the unsaturated soil model theories, load-induced dynamic response in unsaturated soil has been also explored. For 
example, in order to solve concentrated force source problem, the classical Green's functions were derived by Gatmiri and Jabbari [12] in both frequency and time domains using the Kupradze method. Lo [13] described the propagation and attenuation of Rayleigh waves, originated from a loading source, along the free surface of the unsaturated soil layer using the potential functions decomposition. Xu [14] investigated Lamb problem in half-space and obtained the dynamic response of unsaturated soils using integral transform method. Zhang et al. [15], by means of integral transform techniques, proposed Green functions in the transformed domain for an arbitrary internal harmonic loading buried in unsaturated soil.

In general, due to interactions between different media phases, or coupling characteristics of media, the analytical solutions are limited to a few simple cases. Actually, the numerical methods are widely applicable for dynamic response in complicated media. However, during the numerical calculations, it is necessary to evaluate the numerical results, where some accurate analytical solutions are necessary to be sought. By means of Laplace transform, Boer et al. [16] studied the dynamic response of saturated soil column with infinite length and incompressible grains and fluid. Schanz and Cheng [17] presented the dynamic response expression of saturated soil column with infinite length under dynamic load using the integral transformation and convolution quadrature method. These problems can be described in Figure 1. Their work had established a benchmark for numerical calculation solving dynamic response, namely, wave propagation, in saturated media. Note that in the previous theoretical investigations, the results cannot be utilized in the one-dimensional unsaturated media.

Therefore, using analytical methods, the transient dynamic response in a one-dimensional unsaturated soil column under dynamic load excitation is presented in the paper. First, the governing equations based on $u_{s}-p_{f}-p_{a}$ (solid displacement-pore fluid pressure-pore air pressure) form were derived clearly. Then, the analytical solution of an impulse loading boundary condition was deduced in the Laplace domain for the case of one-dimensional soil column in order to provide a benchmark for the corresponding numerical solutions. In view of those results, the solutions in the time domain can be extended for arbitrary loading history using a conventional numerical method. Finally, the effects of different parameters on the dynamic response in unsaturated soil columns were discussed rationally.

\section{Governing Equations}

A partially saturated porous medium is a three-phase material, composing of solid particles, pore fluid, and pore air. Following Zhang et al. [15], by neglecting body forces, the momentum balance equation of unit volume of a threephase porous medium can be expressed as

$$
\sigma_{i j, j}=\bar{\rho}_{s} \ddot{u}_{i}^{s}+\bar{\rho}_{f} \ddot{u}_{i}^{f}+\bar{\rho}_{a} \ddot{u}_{i}^{a},
$$

where $\sigma_{i j}$ is the total stress tensor, $\bar{\rho}_{s}=(1-n) \rho_{s}, \bar{\rho}_{f}=n S_{r} \rho_{f}$, and $\bar{\rho}_{a}=n\left(1-S_{r}\right) \rho_{a}$ are the apparent density for solid, fluid, and air phase, respectively, $n$ is porosity, $S_{r}$ is saturation, and $\rho_{m}(m=s, f, a)$ is the absolute mass density of each individual phase. The variables $u_{i}^{s}, u_{i}^{f}$, and $u_{i}^{a}$ represent the displacement vectors in the solid, fluid, and air phases, respectively. The dot in the superscript represents the differentiation with respect to the time variable $(t)$. Note that repeated indices in the subscript denote the conventional summation.

The relative displacement vectors of fluid and air with respect to solid can be defined as

$$
\begin{aligned}
& w_{i}^{f}=n S_{r}\left(u_{i}^{f}-u_{i}^{s}\right), \\
& w_{i}^{a}=n\left(1-S_{r}\right)\left(u_{i}^{a}-u_{i}^{s}\right) .
\end{aligned}
$$

And the Laplace transform for function $f(t, x, y, z)$ is given by

$$
\mathscr{L}\{f(t)\}=\int_{0}^{+\infty} e^{-s t} f(t) \mathrm{d} t=\tilde{f}(s),
$$

where $s$ is a complex variable.

Substituting equations (2a) and (2b) into equation (1) and performing Laplace transform with the assumption of a stationary initial state, the momentum balance equation in the Laplace domain can be expressed as

$$
\widetilde{\sigma}_{i j, j}=\rho s^{2} \widetilde{u}_{i}^{s}+\rho_{f} s^{2} \widetilde{w}_{i}^{f}+\rho_{a} s^{2} \widetilde{w}_{i}^{a},
$$

where the total density $\rho$ of the solid-fluid-air mixture is determined by

$$
\rho=(1-n) \rho_{s}+n S_{r} \rho_{f}+n\left(1-S_{r}\right) \rho_{a} .
$$

The elastic solid skeleton is assumed to be isotropic and homogeneous. Hence, the constitutive stress-strain equations for the three-phase porous medium is given as

$$
\sigma_{i j}=\lambda e \delta_{i j}+2 \mu \varepsilon_{i j}-\delta_{i j} \alpha p,
$$

where $\lambda$ and $\mu$ are two Lamb's constants, $e=u_{j, j}^{s}$ is the volumetric strain, and $\varepsilon_{i j}$ is the solid strain determined by $\varepsilon_{i j}=(1 / 2)\left(u_{i, j}^{s}+u_{j, i}^{s}\right)$. The parameter $\alpha$ is the effective stress coefficient given by $\alpha=1-K_{b} / K_{s}$ where $K_{b}=\lambda+(2 / 3) \mu$ denotes the bulk modulus of soil skeleton and $K_{s}$ is the compressibility modulus of the solid particles. The variable $p$ represents the mean pore pressure acting on the solid particles, i.e., $p=\gamma p_{f}+(1-\gamma) p_{a}, \gamma$ is a parameter related to the effective stress due to matrix suction, $p_{f}$ and $p_{a}$ are the pore fluid and pore gas pressures, respectively, and $\delta_{i j}$ is Kronecher's delta.

Taking the derivative of equation (6) and applying Laplace transform lead to

$$
\widetilde{\sigma}_{i j, j}=\mu \widetilde{u}_{i, j j}^{s}+(\lambda+\mu) \widetilde{u}_{j, j i}^{s}-\alpha \widetilde{p}_{, i} .
$$

According to equations (4) and (7), the following equation can be obtained: 


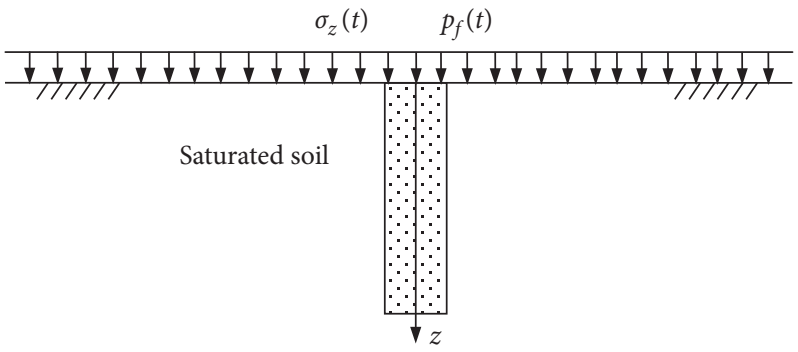

Figure 1: Investigated problem by Boer et al. [16] and Schanz and Cheng [17].

$$
\begin{aligned}
& \mu \widetilde{u}_{i, j j}^{s}+(\lambda+\mu) \widetilde{u}_{j, j i}^{s}-\alpha \widetilde{p}_{, i} \\
& =\rho s^{2} \widetilde{u}_{i}^{s}+\rho_{f} s^{2} \widetilde{w}_{i}^{f}+\rho_{a} s^{2} \widetilde{w}_{i}^{a} .
\end{aligned}
$$

For an isotropic medium, the generalized Darcy's law that is usually adopted to describe the motion of fluid and gas air without considering the body forces is

$$
\begin{array}{r}
n S_{r}\left(\dot{u}_{i}^{f}-\dot{u}_{i}^{s}\right)=\kappa_{f}\left(-p_{f, i}-\rho_{f} \ddot{u}_{i}^{f}\right), \\
n\left(1-S_{r}\right)\left(\dot{u}_{i}^{a}-\dot{u}_{i}^{s}\right)=\kappa_{a}\left(-p_{a, i}-\rho_{a} \ddot{u}_{i}^{a}\right),
\end{array}
$$

where $\kappa_{f}=k_{f} / \eta_{f}, \kappa_{a}=k_{a} / \eta_{a}$ in which $k_{f}$ and $k_{a}$ are the intrinsic permeability of fluid and air, respectively, and $\eta_{f}$ and $\eta_{a}$ are the dynamic viscosity of fluid and air, respectively.

Referring to equations ( $2 \mathrm{a})$ and (2b), the fluid flux $q_{i}^{f}$ and air flux $q_{i}^{a}$ can be defined as

$$
\begin{aligned}
& q_{i}^{f}=n S_{r}\left(\dot{u}_{i}^{f}-\dot{u}_{i}^{s}\right)=\dot{w}_{i}^{f}, \\
& q_{i}^{a}=n S_{r}\left(\dot{u}_{i}^{a}-\dot{u}_{i}^{s}\right)=\dot{w}_{i}^{a} .
\end{aligned}
$$

Substituting equations (10a) and (10b) into equations (9a) and (9b) and combining with equations (2a) and (2b) give

$$
\begin{aligned}
& q_{i}^{f}=-\kappa_{f}\left(p_{f, i}+\frac{\rho_{f}}{n S_{r}} \ddot{w}_{i}^{f}+\rho_{f} \ddot{u}_{i}^{s}\right), \\
& q_{i}^{a}=-\kappa_{a}\left(p_{a, i}+\frac{\rho_{a}}{n\left(1-S_{r}\right)} \ddot{w}_{i}^{a}+\rho_{f} \ddot{u}_{i}^{s}\right) .
\end{aligned}
$$

By applying the Laplace transform defined in equations (3) to (11a) and (11b), the relative displacement vectors of fluid and air with respect to solid can be written as

$$
\begin{aligned}
& \widetilde{w}_{i}^{f}=-\frac{\kappa_{f} n S_{r} \rho_{f} s^{2}}{s n S_{r}+s^{2} \rho_{f} \kappa_{f}} \cdot \frac{1}{\rho_{f} s^{2}}\left(\tilde{p}_{f, i}+\rho_{f} s^{2} \widetilde{u}_{i}^{s}\right) \\
&=-\frac{\beta_{f}}{\rho_{f} s^{2}}\left(\tilde{p}_{f, i}+\rho_{f} s^{2} \widetilde{u}_{i}^{s}\right), \\
& \widetilde{w}_{i}^{a}=-\frac{\kappa_{a} n\left(1-S_{r}\right) \rho_{a} s^{2}}{s n\left(1-S_{r}\right)+s^{2} \rho_{a} \kappa_{a}} \cdot \frac{1}{\rho_{a} s^{2}}\left(\widetilde{p}_{f, i}+\rho_{a} s^{2} \tilde{u}_{i}^{s}\right) \\
&=-\frac{\beta_{a}}{\rho_{a} s^{2}}\left(\widetilde{p}_{f, i}+\rho_{a} s^{2} \widetilde{u}_{i}^{s}\right),
\end{aligned}
$$

where $\quad \beta_{f}=\left(\kappa_{f} n S_{r} \rho_{f} s / n S_{r}+s \rho_{f} \kappa_{f}\right) \quad$ and $\beta_{a}=\left(\kappa_{a} n\left(1-S_{r}\right) \rho_{a} s / n\left(1-S_{r}\right)+s \rho_{a} \kappa_{a}\right)$.

Substituting equations (12a) and (12b) into equation (8) yields

$$
\begin{aligned}
& \mu \widetilde{u}_{i, j j}^{s}+(\lambda+\mu) \widetilde{u}_{j, j i}^{s}-\alpha \widetilde{p}_{, i}+\beta_{f} \widetilde{p}_{f, i}+\beta_{a} \tilde{p}_{a, i} \\
& \quad=s^{2}\left(\rho-\beta_{f} \rho_{f}-\beta_{a} \rho_{a}\right) \tilde{u}_{i}^{s} .
\end{aligned}
$$

Applying the Laplace transform to the mean pore pressure $p$ and taking the derivative lead to

$$
\widetilde{p}_{, i}=\gamma \widetilde{p}_{f, i}+(1-\gamma) \widetilde{p}_{a, i} .
$$

Then, substituting equations (14) into (13) results in

$$
\begin{aligned}
& \mu \widetilde{u}_{i, j j}^{s}+(\lambda+\mu) \widetilde{u}_{j, j i}^{s}+\left(\beta_{f}-\alpha \gamma\right) \tilde{p}_{f, i} \\
& \quad-\alpha(1-\gamma) \widetilde{p}_{a, i}=s^{2} \rho_{0} \widetilde{u}_{i}^{s},
\end{aligned}
$$

where $\rho_{0}=\rho-\beta_{f} \rho_{f}-\beta_{a} \rho_{a}$.

Neglecting the mass exchange between any two phases, the mass balance of a multiphase medium can be expressed as

$$
\frac{\partial \bar{\rho}_{m}}{\partial t}+\left(\bar{\rho}_{m} \dot{u}_{i}^{m}\right)_{, i}=0, \quad(m=s, f, a) .
$$

Based on the soil-water retention curve proposed by Van Genuchten [18], i.e., $S_{e}=\left[1+\left(\chi p_{c}\right)^{d}\right]^{-m}$, the saturation variable $\left(S_{r}\right)$ can be further described as

$$
S_{r}=-\chi m \quad d\left(1-S_{w 0}\right) S_{e}^{(m+1) / m}\left(S_{e}^{-1 / m}-1\right)^{(d-1) / d}\left(p_{a}-p_{f}\right),
$$

where $p_{c}=p_{a}-p_{f}$ is the matrix suction, $S_{e}$ is the effective water saturation given as $S_{e}=S_{e}=\left(S_{r}-S_{w 0}\right) /\left(1-S_{w 0}\right), S_{w 0}$ is the irreducible saturation, and $\chi, m$, and $d$ are the material parameters of the $\mathrm{V}-\mathrm{G}$ model [18].

Combining equations (16) and (17), the following simplified equations can be derived:

$$
\begin{array}{r}
A_{11} \dot{p}_{f}+A_{12} \dot{p}_{a}+A_{13} \dot{u}_{i, i}^{s}+A_{14} \dot{u}_{i, i}^{f}+A_{15} \dot{u}_{i, i}^{a}=0, \\
A_{21} \dot{p}_{f}+A_{22} \dot{p}_{a}+A_{24} \dot{u}_{i, i}^{f}+A_{25} \dot{u}_{i, i}^{a}=0,
\end{array}
$$

where $A_{11} \sim A_{25}$ are coefficients which are given in equation (A.1).

According to equations (18a) and (18b), $p_{f}$ and $p_{a}$ can be found as 


$$
\begin{aligned}
& -p_{f}=a_{11} u_{i, i}^{s}+a_{12} u_{i, i}^{f}+a_{13} u_{i, i}^{a}, \\
& -p_{a}=a_{21} u_{i, i}^{s}+a_{22} u_{i, i}^{f}+a_{23} u_{i, i}^{a},
\end{aligned}
$$

where the coefficients $a_{11} \sim a_{23}$ are presented in equation (A.2).

Taking the derivative of equations (19a) and (19b) and combining with equations (2a) and (2b) lead to

$$
\begin{aligned}
& -p_{f, i}=D_{1} u_{j, j i}^{s}+D_{2} w_{j, j i}^{f}+D_{3} w_{j, j i}^{a}, \\
& -p_{a, i}=D_{4} u_{j, j i}^{s}+D_{5} w_{j, j i}^{f}+D_{6} w_{j, j i}^{a},
\end{aligned}
$$

where the coefficients $D_{1} \sim D_{6}$ are defined in equation (A.3).

Applying the Laplace transform into equations (20a) and (20b) and combining with equations (12a) and (12b) result in

$$
\begin{aligned}
& \left(D_{2} \frac{\beta_{f}}{\rho_{f} s^{2}}+D_{3} \frac{\beta_{a}}{\rho_{a} s^{2}}\right) \tilde{p}_{f, i i}-\widetilde{p}_{f}-\left(D_{1}-D_{2} \beta_{f}-D_{3} \beta_{a}\right) \widetilde{u}_{i, i}^{s}=0 \\
& \left(D_{5} \frac{\beta_{f}}{\rho_{f} s^{2}}+D_{6} \frac{\beta_{a}}{\rho_{a} s^{2}}\right) \tilde{p}_{a, i i}-\widetilde{p}_{a}-\left(D_{4}-D_{5} \beta_{f}-D_{6} \beta_{a}\right) \widetilde{u}_{i, i}^{s}=0 .
\end{aligned}
$$

Therefore, the governing equations of motion for unsaturated soil with $u_{s}-p_{f}-p_{a}$ (solid displacement-pore fluid pressure-pore air pressure) form in the Laplace domain are obtained as equations (15), (21a), and (21b).

\section{Analytical Solutions of One-Dimensional Wave Propagation}

3.1. General Solutions. A one-dimensional unsaturated porous soil column under dynamic loading is illustrated in Figure 2, where the length of the column, measured in the vertical $(z)$ direction, is represented by $L$. The side and the base of the column are assumed to be rigid, frictionless, and impermeable. The vertical normal stress $\sigma_{z}$ is applied at the top of the column. The vertical displacement $\left(u_{z}\right)$, fluid pressure $\left(p_{f}\right)$, and air pressure $\left(p_{a}\right)$ in the column are still unknown, and their directions at the top boundary are assumed to be normal to the boundary along the vertical $(z)$ direction. The loading function $f(t)$ is an arbitrary temporal function that describes the surface loading acting on the solid skeleton, and $P_{0}$ denotes the loading amplitude. For the one-dimensional loading scenario, the governing equations, equations (15), (21a), and (21b), can be expressed as

$$
(\lambda+2 \mu) \widetilde{u}_{z, z z}+\left(\beta_{f}-\alpha \gamma\right) \tilde{p}_{f, z}+\left(\beta_{a}-\alpha(1-\gamma)\right) \widetilde{p}_{a, z}=s^{2} \rho_{0} \widetilde{u}_{z}^{s},
$$

$$
\left(D_{2} \frac{\beta_{f}}{\rho_{f}}+D_{3} \frac{\beta_{a}}{\rho_{a}}\right) \frac{1}{s^{2}} \widetilde{p}_{f, z z}-\widetilde{p}_{f}-\left(D_{1}-D_{2} \beta_{f}-D_{3} \beta_{a}\right) \tilde{u}_{z, z}^{s}=0,
$$

$$
\left(D_{5} \frac{\beta_{f}}{\rho_{f}}+D_{6} \frac{\beta_{a}}{\rho_{a}}\right) \frac{1}{s^{2}} \widetilde{p}_{a, z z}-\widetilde{p}_{a}-\left(D_{4}-D_{5} \beta_{f}-D_{6} \beta_{a}\right) \widetilde{u}_{z, z}^{s}=0 .
$$

And equations (22a), (22b), and (22c) can be rewritten as

$$
\begin{aligned}
E_{11} \tilde{u}_{z, z z}^{s}+E_{12} \widetilde{p}_{f, z}+E_{13} \widetilde{p}_{a, z}-s^{2} \rho_{0} \widetilde{u}_{z}^{s}=0, \\
E_{22} \widetilde{p}_{f, z z}-\widetilde{p}_{f}-E_{21} \widetilde{u}_{z, z}^{s}=0, \\
E_{33} \widetilde{p}_{a, z z}-\widetilde{p}_{a}-E_{31} \tilde{u}_{z, z}^{s}=0,
\end{aligned}
$$

where the coefficients $E_{11} \sim E_{33}$ are defined in equation (A.4).

Note that equations (23a), (23b), and (23c) can be solved as

$$
\begin{aligned}
& \tilde{u}_{z}^{s}=U e^{\xi_{s z}} \\
& \widetilde{p}_{f}=P_{f} e^{\xi_{s z}} \\
& \tilde{p}_{a}=P_{a} e^{\xi_{s z}}
\end{aligned}
$$

Substituting equation (24) into equations (23a), (23b), and $(23 \mathrm{c})$ leads to an eigenvalue problem in terms of $\xi$ as

$$
\left[\begin{array}{ccc}
E_{11} \xi^{2}-\rho_{0} & E_{12} \frac{\xi}{s} & E_{13} \frac{\xi}{s} \\
E_{21} \xi s & 1-E_{22} \xi^{2} s^{2} & 0 \\
E_{31} \xi s & 0 & 1-E_{33} \xi^{2} s^{2}
\end{array}\right] \cdot\left[\begin{array}{c}
U \\
P_{f} \\
P_{a}
\end{array}\right]=0 .
$$

The corresponding characteristic equation is

$$
B_{3} r^{3}+B_{2} r^{2}+B_{1} r-\rho_{0}=0,
$$

where $B_{1} \sim B_{3}$ and $r$ can be found in equation (A.5).

Providing that equation (26) yielded three roots, i.e., $r_{i}(i=1 \sim 3)$, the eigenvalue problem would have six complex roots $\pm \xi_{n}(n=1,2,3)\left(\operatorname{Re}\left(\xi_{n}\right) \geq 0\right)$ because of the square root operation. Therefore, the complete solution of equations (23a), (23b), and (23c) given by 


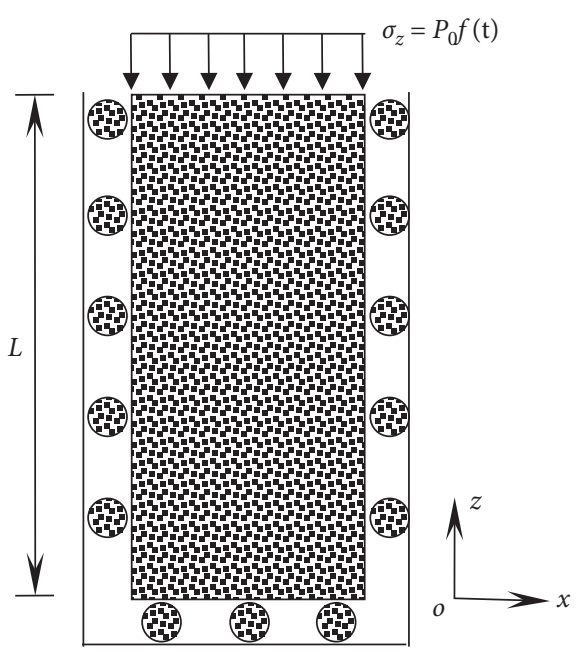

FIGURE 2: Schematic of a one-dimensional unsaturated soil column under dynamic loading.

$$
\begin{aligned}
& \widetilde{u}_{z}^{s}=C_{i} e^{\xi_{i} s z}, \\
& \widetilde{p}_{f}=\vartheta_{i} C_{i} e^{\xi_{i} s z} s, \\
& \widetilde{p}_{a}=\zeta_{i} C_{i} e^{\xi_{i} s z} s, \quad(i=1 \sim 6),
\end{aligned}
$$

where $\vartheta_{i}=\left(E_{21} \xi_{i} / E_{22} \xi_{i}^{2} s^{2}-1\right), \quad \zeta_{i}=\left(E_{31} \xi_{i} / E_{33} \xi_{i}^{2} s^{2}-1\right)$, $\xi_{1}=-\xi_{4}, \xi_{2}=-\xi_{5}, \xi_{3}=-\xi_{6}, C_{i}$ are a series of constants that need to be determined. The eigenvector relations derived from equation (25) are expressed as

$$
\begin{aligned}
& P_{f}=\frac{E_{21} \xi s}{E_{22} \xi^{2} s^{2}-1} U=\vartheta s U, \\
& P_{a}=\frac{E_{31} \xi s}{E_{33} \xi^{2} s^{2}-1} U=\zeta s U .
\end{aligned}
$$

3.2. Boundary Conditions. The solutions to the displacement and pressure in equation (27) involve unknown constants $\left(C_{i}\right)$ which can be determined by the boundary conditions. As aforementioned, the top boundary of the column is permeable for both the fluid and the air. The impulse function $f(t)=\delta(t)$ is adopted herein where $\delta(t)$ represents the Dirac distribution. Therefore, the boundary conditions are given as

$$
\begin{aligned}
& \tilde{p}_{f}(z=L)=0, \\
& \tilde{p}_{a}(z=L)=0, \\
& \tilde{\sigma}_{z}(z=L)=-P_{0}, \\
& \tilde{u}_{z}(z=0)=0, \\
& \tilde{q}_{f}(z=0)=0, \\
& \tilde{q}_{a}(z=0)=0 .
\end{aligned}
$$

Substituting equation (27) into the one-dimensional constitutive relation equation (6) yields

$$
\begin{aligned}
\widetilde{\sigma}_{z} & =(\lambda+2 \mu) \widetilde{u}_{z, z}-\alpha \widetilde{p} \\
& =(\lambda+2 \mu) \xi_{i} s C_{i} e^{\xi_{i} s z}-\alpha \gamma \widetilde{p}_{f}-\alpha(1-\gamma) \widetilde{p}_{a} .
\end{aligned}
$$

Combining equation (27) with equations (12a) and (12b) gives the following relations:

$$
\begin{aligned}
& \tilde{q}_{f}=-\frac{\beta_{f}}{\rho_{f} s}\left(\vartheta_{i} C_{i} \xi_{i} s^{2} e^{\xi_{i} s z}+\rho_{f} s^{2} \widetilde{u}_{z}^{s}\right), \\
& \tilde{q}_{a}=-\frac{\beta_{a}}{\rho_{a} s}\left(\zeta_{i} C_{i} \xi_{i} s^{2} e^{\xi_{i} s z}+\rho_{a} s^{2} \widetilde{u}_{z}^{s}\right) .
\end{aligned}
$$

Accordingly, a series of linear equations with respect to the unknown constants $\left(C_{i}\right)$ can be derived by substituting equations (30), (31a), and (31b) into equation (29). By substituting the calculated $C_{i}$ into equation (27), the solutions for the vertical displacement of solid and pressures of fluid and air in the column can then be derived in the Laplace domain as

$$
\begin{aligned}
& \tilde{\mathcal{u}}_{z}=\frac{P_{0}}{E_{0} s} \psi_{i} \sec \quad h\left(s L \xi_{i}\right) \sinh \left(s z \xi_{i}\right), \\
& \tilde{p}_{f}=\frac{P_{0}}{E_{0}} \psi_{i} \sec \quad h\left(s L \xi_{i}\right) \cosh \left(s z \xi_{i}\right) \vartheta_{i}, \\
& \tilde{p}_{a}=\frac{P_{0}}{E_{0}} \psi_{i} \sec \quad h\left(s L \xi_{i}\right) \cosh \left(s z \xi_{i}\right) \zeta_{i},
\end{aligned}
$$

where $E_{0}, \delta_{i}$, and $\psi_{i}(i=1 \sim 3)$ are defined in equation (A.6).

The response in the time domain can also be calculated using the convolution integral. Therefore, the vertical displacement, and pressures of fluid and air can be derived as

$$
\begin{aligned}
u_{z} & =\int_{0}^{t} \mathscr{L}^{-1}\left\{\tilde{u}_{z}(s, z)\right\}(\tau, z) f(t-\tau) \mathrm{d} \tau, \\
p_{f} & =\int_{0}^{t} \mathscr{L}^{-1}\left\{\widetilde{p}_{f}(s, z)\right\}(\tau, z) f(t-\tau) \mathrm{d} \tau,
\end{aligned}
$$




$$
p_{a}=\int_{0}^{t} \mathscr{L}^{-1}\left\{\widetilde{p}_{a}(s, z)\right\}(\tau, z) f(t-\tau) \mathrm{d} \tau,
$$

where $\mathscr{L}^{-1}$ is the inverse Laplace transform operator.

\section{Verification of the Solution}

The solution calculated for unsaturated soil in the previous section was verified using the degenerated solution compared with the results in one-dimensional saturated soil column presented by Schanz and Cheng [17]. For this purpose, the foregoing coefficients $S_{r}, \gamma, \kappa_{a}, \rho_{a}$, and $A_{s}$ are simplified for the saturation state:

$$
\begin{aligned}
S_{r} & =1, \\
\gamma & =1, \\
k_{a} & =0, \\
\rho_{a} & =0, \\
A_{s} & =0 .
\end{aligned}
$$

Also, the coefficients in equations (18a) and (18b) can be rewritten as

$$
\begin{aligned}
& A_{11}=\frac{\alpha-n}{K_{s}}+\frac{n}{K_{f}}=M^{-1}, \\
& A_{13}=\alpha-n, \\
& A_{14}=n, \\
& A_{12}=A_{15}=A_{21}=A_{24}=A_{25}=0 .
\end{aligned}
$$

The coefficients in equations (20a) and (20b) can also be simplified as

$$
\begin{aligned}
& D_{1}=\alpha M, \\
& D_{2}=M, \\
& D_{3}=D_{4}=D_{5}=D_{6}=0 .
\end{aligned}
$$

Therefore, the coefficients in equations (23a), (23b), and (23c) can be rewritten as

$$
\begin{aligned}
\beta_{a} & =0, \\
\rho_{0} & =\rho-\beta_{f} \rho_{f}, \\
E_{12} & =\beta_{f}-\alpha, \\
E_{13} & =0, \\
E_{21} & =\left(\alpha-\beta_{f}\right) M, \\
E_{22} & =\frac{M \beta_{f}}{\left(\rho_{f} s^{2}\right)}, \\
E_{31} & =0, \\
E_{33} & =0 .
\end{aligned}
$$

Accordingly, linear equation (25) is reformed as

$$
\left[\begin{array}{cc}
(\lambda+2 \mu) \xi^{2}-\left(\rho-\beta_{f} \rho_{f}\right) & \left(\beta_{f}-\alpha\right) \frac{\xi}{s} \\
-s\left(\alpha-\beta_{f}\right) \xi & \xi^{2} \frac{\beta_{f}}{\rho_{f}}-\frac{1}{M}
\end{array}\right] \cdot\left[\begin{array}{c}
U \\
P_{f}
\end{array}\right]=0 .
$$

Due to the absence of the air in the saturated soil, the air pressure is equal to zero, i.e., $\widetilde{p}_{a}=0$, and the coefficients defined in equation (28b) satisfy the relation $\zeta_{1}=\zeta_{2}=\zeta_{3}=0$. Finally, the solutions for the vertical displacement of solid and fluid pressure in the saturated scenario can be derived as

$$
\begin{aligned}
& \tilde{u}_{z}=\frac{P_{0}}{(\lambda+2 \mu) s\left(\vartheta_{2} \xi_{1}-\vartheta_{1} \xi_{2}\right)}\left(\vartheta_{1} \operatorname{sech}\left(s L \xi_{2}\right) \sinh \left(s z \xi_{2}\right)-\vartheta_{2} \operatorname{sech}\left(s L \xi_{1}\right) \sinh \left(s z \xi_{1}\right)\right), \\
& \tilde{p}_{f}=\frac{P_{0} \vartheta_{1} \vartheta_{2}}{(\lambda+2 \mu)\left(\vartheta_{2} \xi_{1}-\vartheta_{1} \xi_{2}\right)}\left(\operatorname{sech}\left(s L \xi_{2}\right) \cosh \left(s z \xi_{2}\right)-\operatorname{sech}\left(s L \xi_{1}\right) \cosh \left(s z \xi_{1}\right)\right) .
\end{aligned}
$$

Figure 3 compares the vertical displacement of solid particles and fluid pressure calculated in this study to the solutions of Schanz and Cheng [17]. The values of the unsaturated soil variables in this paper are listed in Table 1 that are taken from Zhang et al. [15]. As can be seen, the results of this study are remarkably consistent with those of Schanz and Cheng [17].

\section{Parametric Study}

In order to investigate the effects of fluid saturation, fluid permeability, and the porosity on the vertical displacement of solid and pressures of fluid and air, a series of parametric studies are conducted. The adopted parameters are given in Table 1, and the boundary conditions are the same as equation (29), and a Heaviside step function with $P_{0}=1$ is assumed for the time history of loading. Figure 4 shows the 


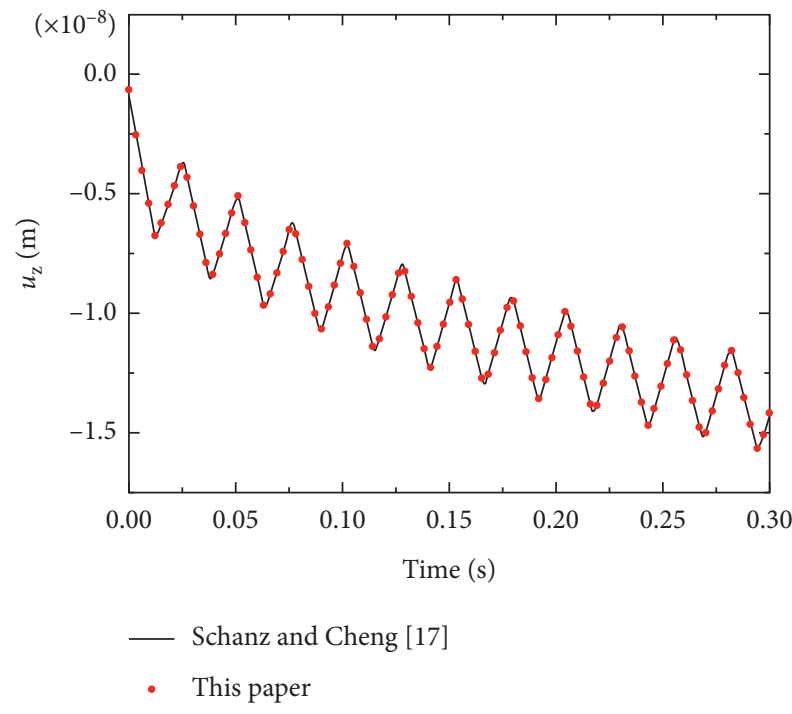

(a)

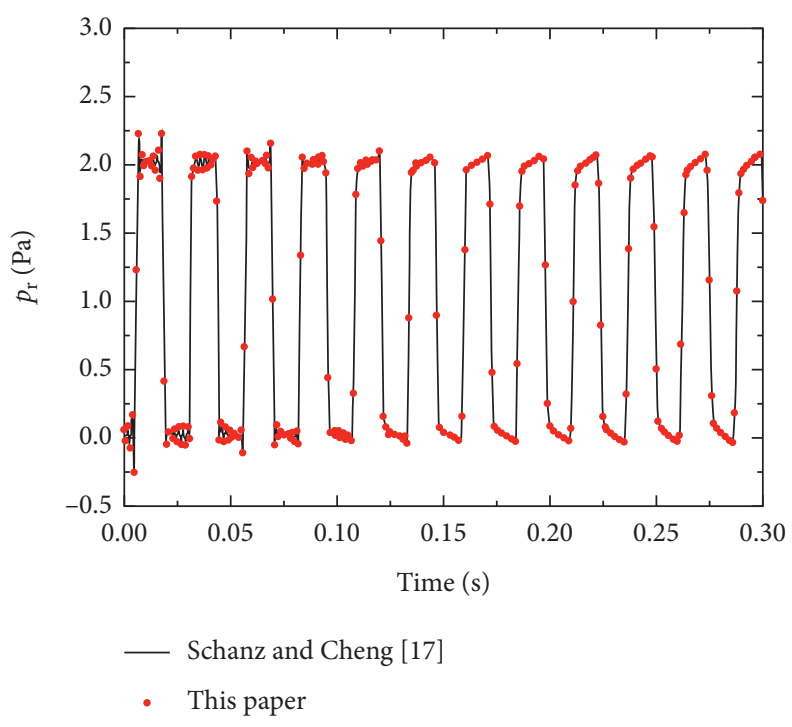

(b)

Figure 3: Distributions of the vertical displacement of solid particles $u_{z}(t, z=L)$ and the fluid pressure $p_{f}(t, z=0)$ calculated in this study compared to those reported by Schanz and Cheng [17]. (a) $u_{z}$. (b) $p_{f}$.

TABLE 1: Unsaturated soil specification.

\begin{tabular}{lcc}
\hline Parameter & Unit & Selected value \\
\hline$K_{s}$ & $\mathrm{~Pa}$ & $3.5 \times 10^{10}$ \\
$\rho_{s}$ & $\mathrm{~kg} / \mathrm{m}^{3}$ & 2650 \\
$K_{f}$ & $\mathrm{~Pa}$ & $2.25 \times 10^{9}$ \\
$\rho_{f}$ & $\mathrm{~kg} / \mathrm{m}^{3}$ & 1000 \\
$\eta_{f}$ & $\mathrm{~Pa} \cdot \mathrm{s}$ & $1 \times 10^{-3}$ \\
$\kappa_{f}$ & $\mathrm{~m}^{2}$ & $5.3 \times 10^{-12}$ \\
$K_{a}$ & $\mathrm{~Pa}$ & $1.45 \times 10^{5}$ \\
$\rho_{a}$ & $\mathrm{~kg} / \mathrm{m}^{3}$ & 1.28 \\
$\eta_{a}$ & $\mathrm{~Pa} \cdot \mathrm{s}$ & $1.8 \times 10^{-7}$ \\
$K_{b}$ & $\mathrm{~Pa}$ & $8.33 \times 10^{6}$ \\
$\mu$ & $\mathrm{Pa}$ & $3.85 \times 10^{6}$ \\
$n$ & - & 0.45 \\
$S_{r}$ & - & $0.35 \sim 1.0$ \\
$\gamma$ & - & $S_{r}$ \\
$S_{w 0}$ & - & 0.06 \\
$\chi$ & $\mathrm{Pa}$ & $1 \times 10^{-4}$ \\
$m$ & - & 0.5 \\
$d$ & - & 2 \\
\hline
\end{tabular}

distributions of the solid vertical displacement, the fluid and air pressures in a time interval from 0 to $0.8 \mathrm{~s}$ for cases with fluid saturation value varying from 0.4 to 0.9 . As can be seen, when the fluid saturation value is less than 0.9 , the vertical solid displacement response for different cases is fairly close to each other whilst when the saturation values reach 0.9 , the amplitude of solid displacement tends to reduce. In contrast, the effects of the fluid saturation on the pressures of fluid and air are much more evident. As shown in Figures 3(b) and $3(c)$, the amplitude of the fluid and air pressure response increases with the increasing fluid saturation value.

Figure 5 illustrates the distributions of the solid displacement, and the fluid and air pressures in the time coordinate for cases with fluid saturation value varying from 0.9 to 0.99 . Clearly, fluid saturation has an influence on the amplitude of the displacement and pressure responses. As the fluid saturation increases, the amplitude of displacement decreases and pressure responses increase. These responses have the phenomenon of shortening the cycle of periodic change, that is, the periodic change frequency is increasing. This indicates that the wave travels faster.

Figure 6 shows the distributions of the solid displacement, and the fluid and air pressures with time for cases with fluid intrinsic permeability value varying from $10^{-13}$ to $10^{-10}$. Clearly, the magnitude of the intrinsic permeability $\left(\kappa_{f}\right)$ has little impact on the vertical displacement of solid $\left(u_{\mathrm{z}}\right)$, the fluid pressure $\left(p_{f}\right)$, and the air pressure $\left(p_{a}\right)$, except for the case with $\kappa_{f}$ equal to $10^{-9}$ which manifests slight deviation from the other cases when the magnitude of the displacement and pressures approach the maximum and minimum values.

Figure 7 shows the distributions of the solid displacement, and the fluid and air pressures with time for cases with porosity value varying from 0.35 to 0.55 . It can be seen that the increase in porosity $(n)$ insignificantly affects the amplitude of the solid displacements $\left(u_{z}\right)$ but it will result in a slight increase in the frequency. On the other hand, as the porosity increases, the amplitude of the fluid and air pressures will decrease accordingly while the corresponding frequency will increase. 

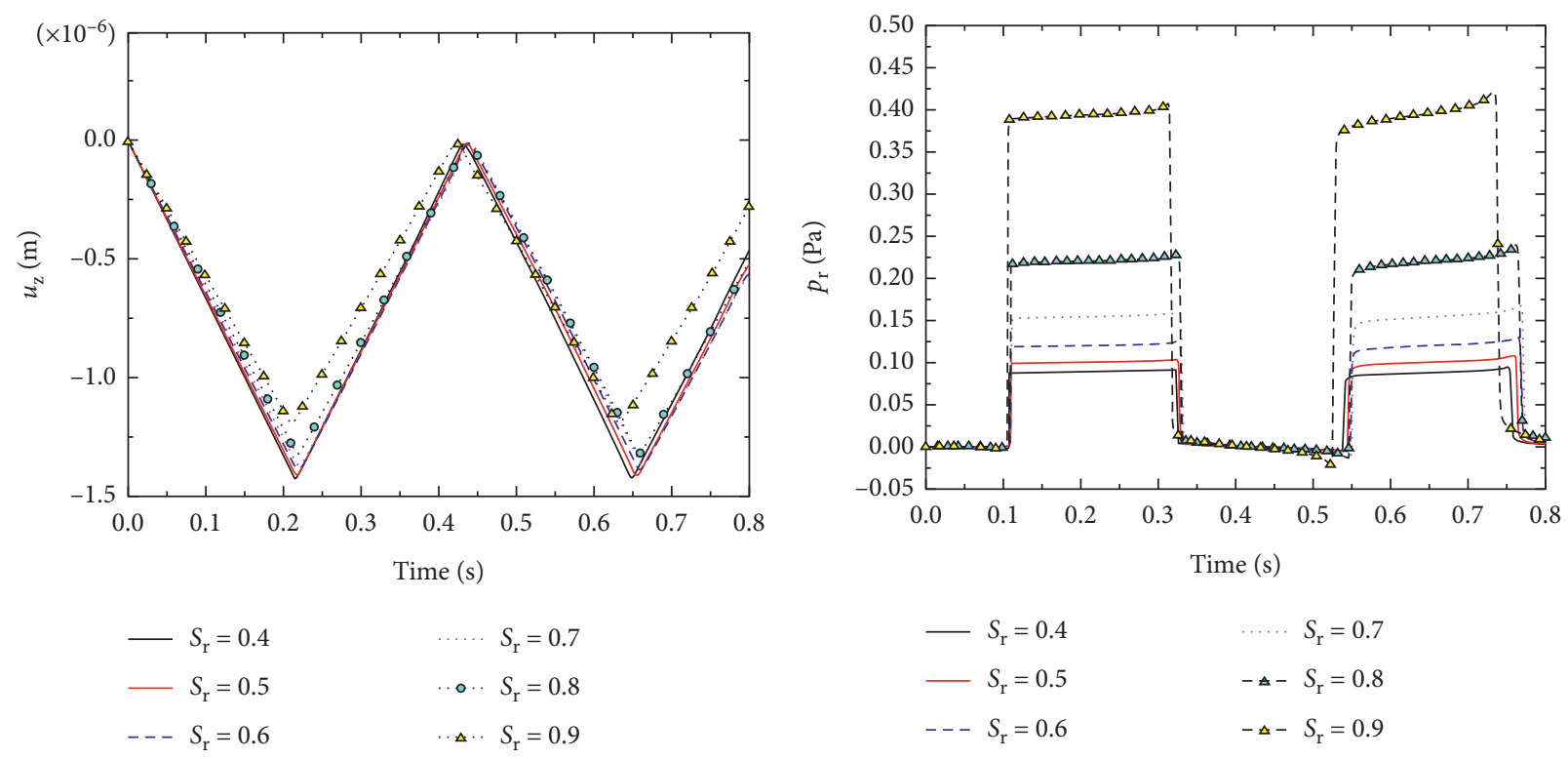

$$
\begin{aligned}
& \ldots \cdots S_{r}=0.7 \\
& \therefore \circ \quad S_{r}=0.8 \\
& \therefore \quad S_{r}=0.9
\end{aligned}
$$

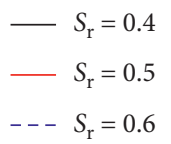

(a)

$$
\begin{aligned}
& \ldots . . S_{r}=0.7 \\
& -\Delta-S_{r}=0.8 \\
& -\Delta-S_{r}=0.9
\end{aligned}
$$

(b)

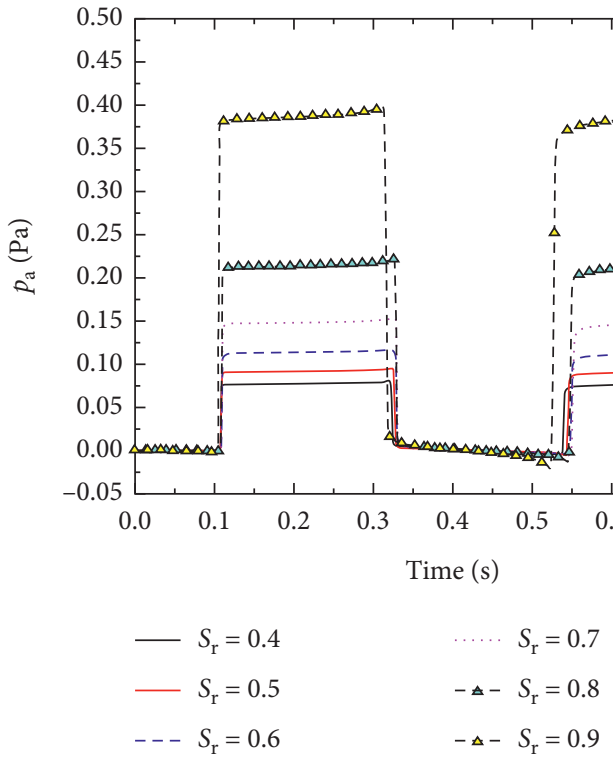

(c)

Figure 4: Distributions of the displacement of solid particles $u_{z}(t, z=L)$, fluid pressure $p_{f}(t, z=0)$, and air pressure $p_{a}(t, z=0)$ with time using different fluid saturation values $\left(S_{r}=0.4\right.$ to 0.9$)$. (a) $u_{z}$. (b) $p_{f}$. (c) $p_{a}$.

\section{Summary and Conclusions}

This paper studies the dynamic response caused by an impulse load in unsaturated poroelastic materials for the case of onedimensional space using the analytical method. The governing equations in the form of $u_{s}-p_{f}-p_{a}$ (solid displacement-pore fluid pressure-pore air pressure) were derived, and the analytical solutions for a one-dimensional poroelastic column in the Laplace transform domain were calculated, which were transformed to the time domain via the inverse Laplace transform using a numerical method. The solution has a concise closed form. The solutions were verified by comparing to the degeneration results for saturated soil with those reported by Schanz and Cheng [17]; and the effects of three important parameters, i.e., fluid saturation, fluid permeability, and porosity, were investigated.

From the present parametric analysis, some findings of this study may be summarized as follows:

(i) The increase in fluid saturation $\left(S_{r}\right)$ larger than 0.9 or porosity $(n)$ has an evident effect on the amplitude of the fluid and air pressures as well as the wave velocity

(ii) The amplitude of solid displacement $\left(u_{z}\right)$ gradually decreases when the fluid saturation $\left(S_{r}\right)$ increases. In 

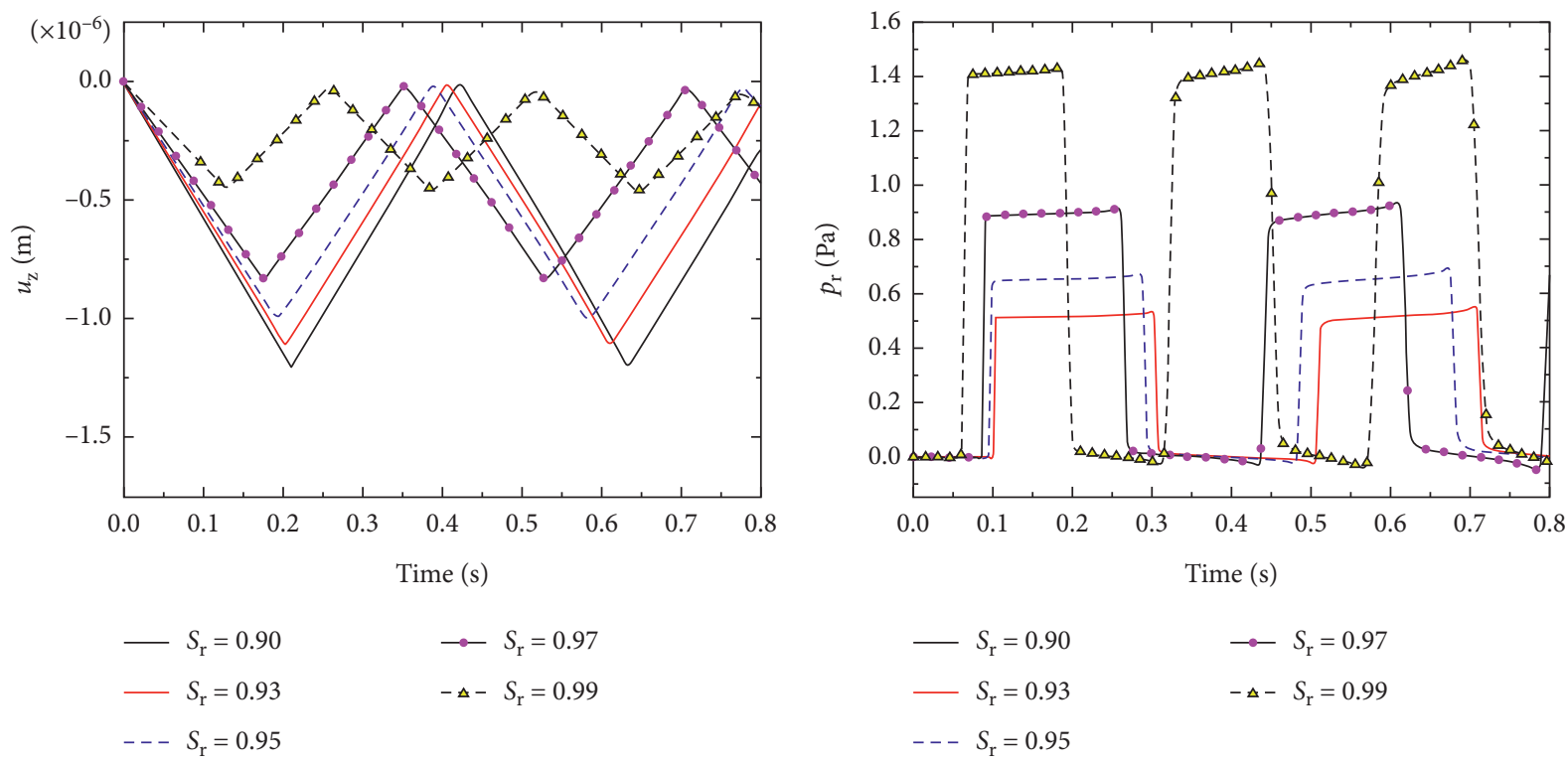

(a)

(b)

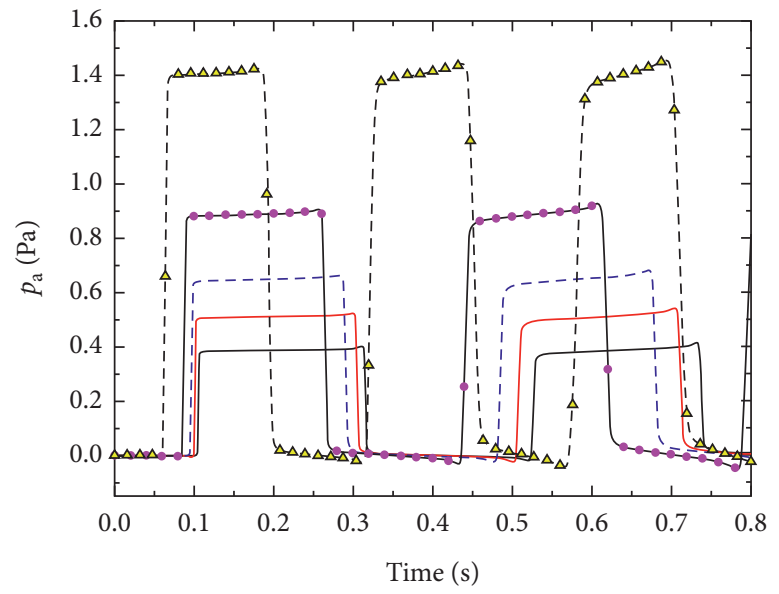

$$
\begin{array}{ll}
-S_{\mathrm{r}}=0.90 & \rightarrow-S_{\mathrm{r}}=0.97 \\
-S_{\mathrm{r}}=0.93 & -\Delta-S_{\mathrm{r}}=0.99 \\
--S_{\mathrm{r}}=0.95 &
\end{array}
$$

(c)

FIGURE 5: Distributions of the vertical displacement of solid particles $u_{z}(t, z=L)$, fluid pressure $p_{f}(t, z=0)$, and air pressure $p_{a}(t, z=0)$ with time using different fluid saturation values $\left(S_{r}=0.9\right.$ to 0.99$)$. (a) $u_{z}$. (b) $p_{f}$. (c) $p_{a}$.

contrast, the amplitude of the fluid and air pressure response increases

(iii) The fluid saturation $\left(S_{r}\right)$ increasing above 0.9 results in wave travels faster significantly

(iv) The variation of the fluid intrinsic permeability $\left(\kappa_{f}\right)$ has little influence on the soil dynamic response until it reaches a high order of magnitude

Note that this study only considered the permeable boundary conditions for both the fluid and air phases, and further research on different types of boundary conditions seems necessary. The paper only discusses a type of unsaturated soil, without considering other practical case. The conclusions have yet to be thoroughly verified. However, for the unsaturated poroelastic soil, by means of the solutions found in this study, the accuracy of different numerical methods applied to the dynamic problem can be evaluated directly $[19,20]$. 

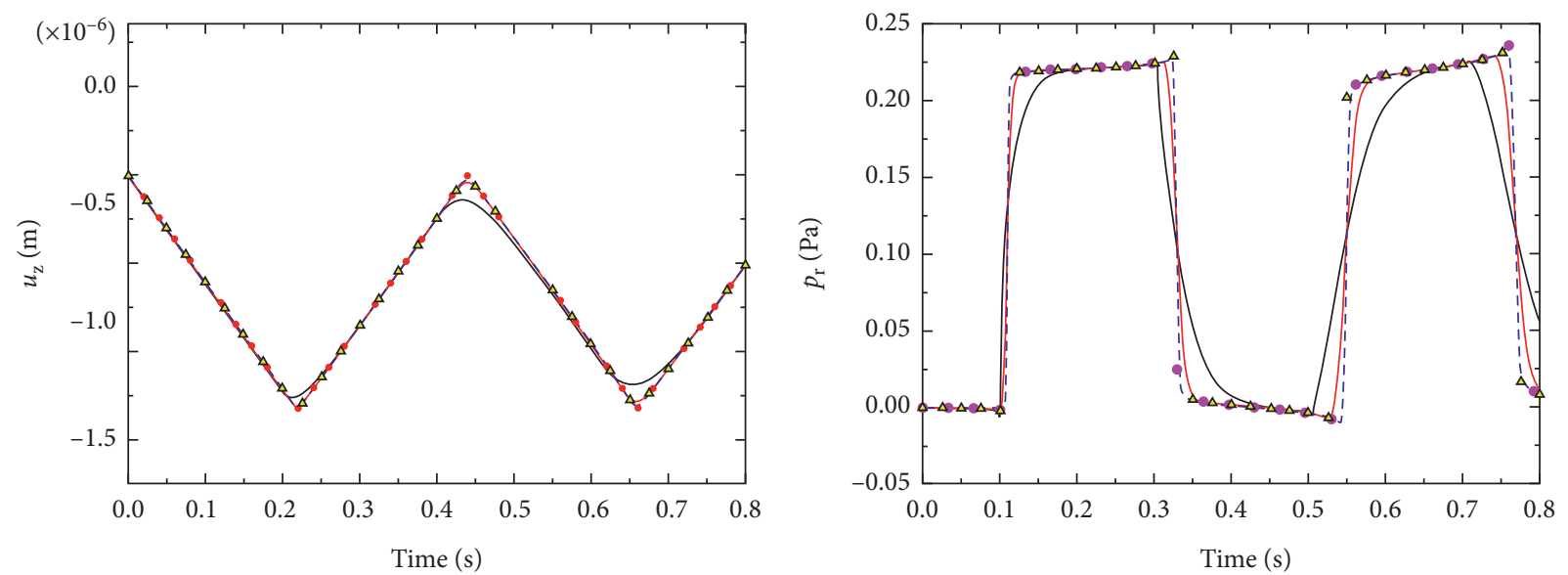

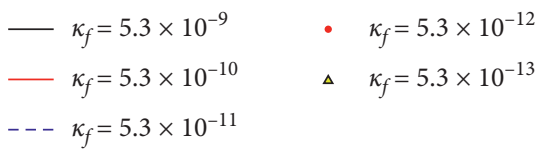

(a)

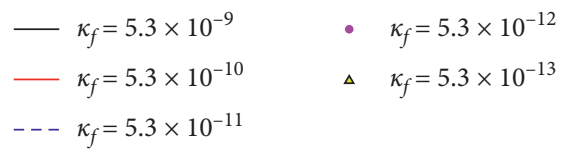

(b)

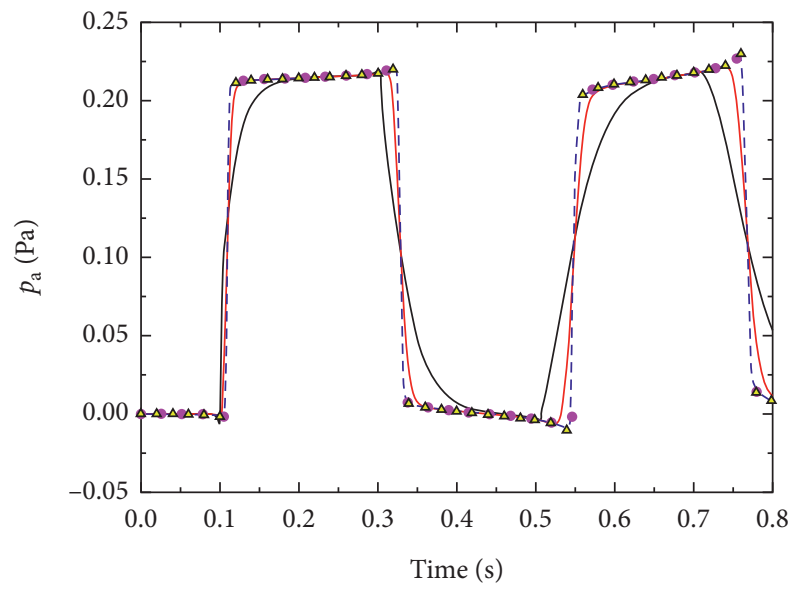

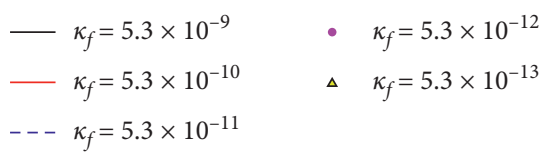

(c)

FiguRe 6: Distributions of the displacement of solid particles $u_{z}(t, z=L)$, fluid pressure $p_{f}(t, z=0)$, and air pressure $p_{a}(t, z=0)$ with time using different fluid permeability values $\left(\kappa_{f}=5.3 \times 10^{-9}\right.$ to $\left.5.3 \times 10^{-13}\right)$. (a) $u_{z}$. (b) $p_{f}$. (c) $p_{a}$. 

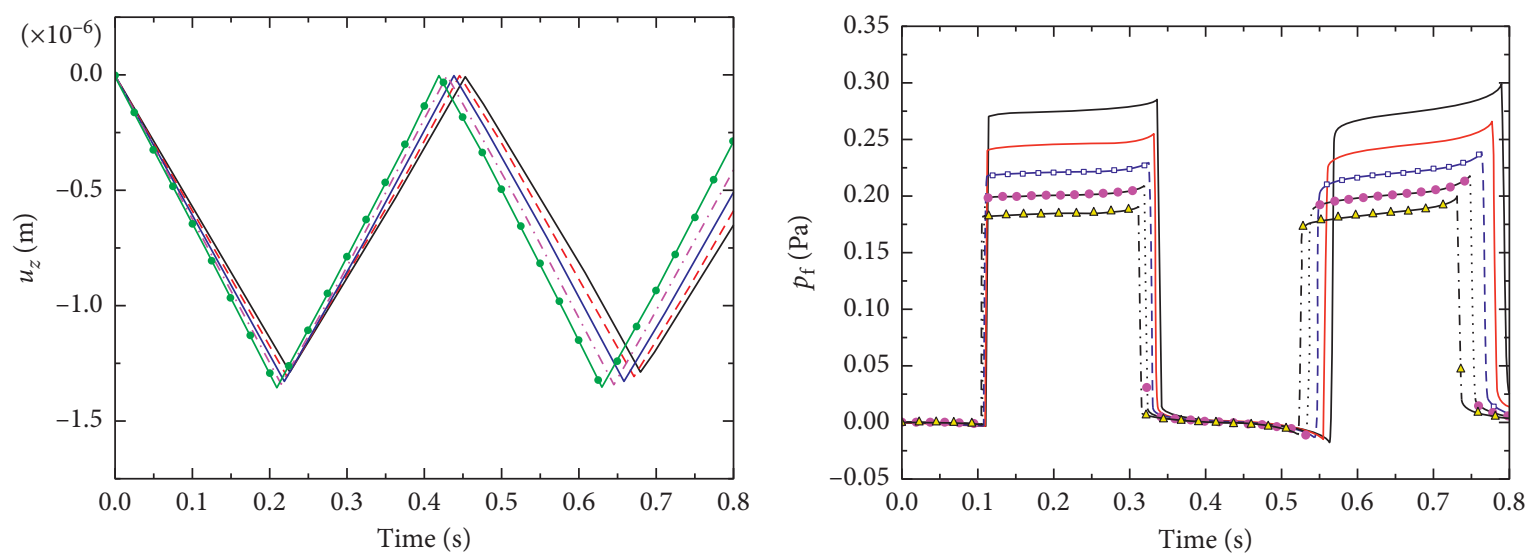

$$
\begin{aligned}
& \text { - } n=0.35 \quad \cdots n=0.50 \\
& --n=0.40 \\
& \longrightarrow n=0.55
\end{aligned}
$$

(a)

(b)

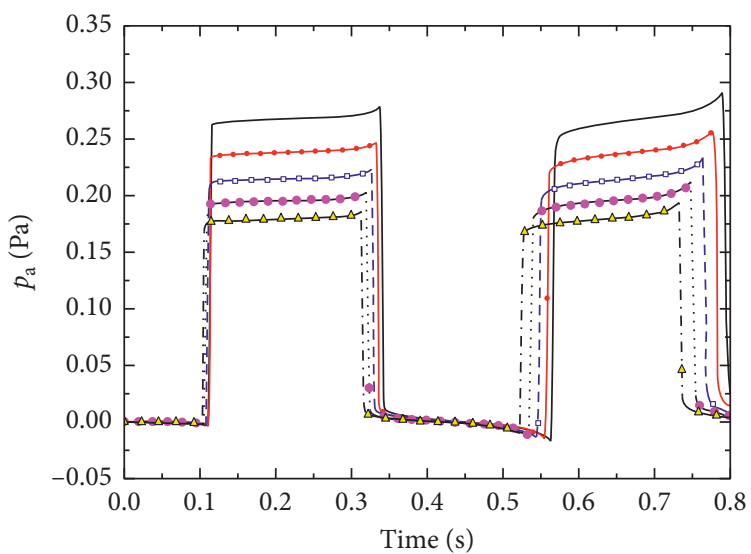

(c)

FiguRe 7: Distributions of the displacement of solid particles $u_{z}(t, z=L)$, fluid pressure $p_{f}(t, z=0)$, and air pressure $p_{a}(t, z=0)$ with time using different porosity values $(n=0.35$ to 0.55$)$. (a) $u_{z}$. (b) $p_{f}$. (c) $p_{a}$.

\section{Notations}

\section{Upper case}

$A_{11}, A_{12}, \ldots, A_{24}$, Calculation coefficients

$A_{s}, A_{0}$ :

$B_{1}, B_{2}, B_{3}$ :

Calculation coefficients

$D_{1}, \ldots, D_{6}, D_{f}, D_{a}$ : Calculation coefficients

$E_{11}, E_{12}, \ldots, E_{33}: \quad$ Calculation coefficients

$E_{0}$ :

$K_{b}$ :

$K_{f}, K_{s}:$

$L:$

$M$ :

$P_{0}$ :

$S_{r}:$

$S_{e}:$

$S_{w 0}$ :

$U, P_{f}, P_{\mathrm{a}}$ :
Calculation coefficient

Bulk modulus of soil skeleton

Compressibility modulus of the fluid and solid grains, respectively

Column length

$1 / A_{11}$

Loading amplitude

Fluid saturation

Effective water saturation

Irreducible saturation

Amplitude vector of solid displacement, fluid, and air, respectively

\section{Lower case}

$a_{11}, a_{12}, \ldots, a_{23}$ : Calculation coefficients

$e$ :

$g$ :

$k_{f}, k_{a}:$

$m, d$ :

$n:$

$p:$

$p_{c}:$

$p_{f}, p_{a}:$

$q_{i}^{f}, q_{i}^{a}:$

$s$ :

$u_{i}^{s}, u_{i}^{f}, u_{i}^{a}:$

$w_{f}, w_{a}:$

$z$ :
Volumetric strain of solid grains

Gravity acceleration

Intrinsic permeability of fluid and air, respectively

V-G model parameter

Porosity

Mean pore pressure

Matrix suction

Pore fluid pressure and air pressure, respectively

Fluid flux and air flux, respectively

Complex variable in the Laplace

transformed domain

Components of displacements vector of solid grains, fluid, and air, respectively Relative displacement vectors of fluid and air, respectively

Coordinate in vertical 
Greek letters

$\alpha: \quad$ Effective stress coefficient

$\beta_{f}, \beta_{a}: \quad$ Calculation coefficients

$\gamma: \quad$ Effective stress parameter

$\delta_{i}: \quad$ Calculation coefficient

$\delta_{i j}: \quad$ Kronecher's delta

$\eta_{f}, \eta_{a}: \quad$ Dynamic viscosity of fluid and air, respectively

$\kappa_{f}, \kappa_{a}: \quad k_{f} / \eta_{f}, k_{a} / \eta_{a}$, respectively

$\lambda, \mu: \quad$ Lamè constants

$\xi: \quad$ Root for characteristic equation

$\rho: \quad$ Bulk density of solid-fluid-gas mixture

$\rho_{0}: \quad \rho \beta_{f f} \rho_{f^{-}} \beta_{a} \rho_{a}$

$\rho_{s}, \rho_{f}, \rho_{a}$ : Absolute mass densities for solid, fluid, and gas, respectively

$\bar{\rho}_{s}, \bar{\rho}_{f}, \bar{\rho}_{a}$ : Apparent density for solid, fluid, and gas phases, respectively

$\sigma_{i j}: \quad$ Total stress tensor

$\vartheta_{i}, \zeta_{i}: \quad$ Calculation coefficients

$\chi$ : $\quad$ V-G model parameter

$\psi_{i}$ : Calculation coefficient.

\section{Appendix}

$$
\begin{aligned}
& A_{11}= \frac{\left(\alpha \gamma-n S_{r}\right)}{K_{s}+n S_{r} / K_{f}}, \\
& A_{12}= \frac{\left(\alpha(1-\gamma)-n\left(1-S_{r}\right)\right)}{K_{s}+n\left(1-S_{r}\right) / K_{a}}, \\
& A_{13}= \alpha-n \\
& A_{14}= n S_{r}, \\
& A_{15}= n\left(1-S_{r}\right) \\
& A_{21}= A_{s}-S_{r}\left(1-S_{r}\right) / K_{f}, \\
& A_{22}= S_{r}\left(1-S_{r}\right) / K_{a}-A_{s}, \\
& A_{24}=-A_{25} \\
& A_{s}=-\chi m \quad d\left(1-S_{w 0}\right) S_{e}^{(m+1) / m}\left(S_{e}^{-1 / m}-1\right)(d-1) / d \\
& a_{11}= \frac{\left(A_{22} A_{13}\right)}{A_{0}}, \\
& a_{22}= \frac{\left(A_{22} A_{14}-A_{12} A_{24}\right)}{A_{0}}, \\
& a_{12}= \frac{\left(A_{11} A_{24}-A_{21} A_{14}\right)}{A_{0}}, \\
& a_{13}= \frac{\left(A_{22} A_{15}-A_{12} A_{25}\right)}{A_{0}}, \\
& a_{21}=\left(A_{21} A_{13}\right) \\
& A_{0} A_{22}-A_{12} A_{21} . \\
& A_{0}
\end{aligned}
$$

$$
\begin{aligned}
& D_{1}=\frac{\left(\alpha A_{22}\right)}{A_{0}}, \\
& D_{2}=\frac{\left(A_{22} A_{14}-A_{12} A_{24}\right)}{\left(A_{14} A_{0}\right)}, \\
& D_{3}=\frac{\left(A_{22} A_{15}-A_{12} A_{25}\right)}{\left(A_{15} A_{0}\right)}, \\
& D_{4}=\frac{\left(-\alpha A_{21}\right)}{A_{0}}, \\
& D_{5}=\frac{\left(A_{11} A_{24}-A_{21} A_{14}\right)}{\left(A_{14} A_{0}\right)}, \\
& D_{6}=\frac{\left(A_{11} A_{25}-A_{21} A_{15}\right)}{\left(A_{15} A_{0}\right)} .
\end{aligned}
$$

$B_{1}=\rho_{0}\left(D_{f}+D_{a}\right)$

$-E_{13} E_{31}-E_{12} E_{21}+E_{11}$,

$\xi^{2}=r$.

$$
\begin{aligned}
E_{0} & =E_{11}\left(\delta_{1}+\delta_{2}+\delta_{3}\right), \\
\delta_{1} & =\vartheta_{1}\left(\zeta_{2} \xi_{3}-\zeta_{3} \xi_{2}\right), \\
\delta_{2} & =\vartheta_{2}\left(\zeta_{3} \xi_{1}-\zeta_{1} \xi_{3}\right), \\
\delta_{3} & =\vartheta_{3}\left(\zeta_{1} \xi_{2}-\zeta_{2} \xi_{1}\right), \\
\psi_{1} & =\vartheta_{3} \zeta_{2}-\vartheta_{2} \zeta_{3}, \\
\psi_{2} & =\vartheta_{1} \zeta_{3}-\vartheta_{3} \zeta_{1}, \\
\psi_{3} & =\vartheta_{2} \zeta_{1}-\vartheta_{1} \zeta_{2} .
\end{aligned}
$$

\section{Data Availability}

The data used to support the findings of this study are available from the corresponding author upon request.

\section{Conflicts of Interest}

The authors declare that there are no conflicts of interest regarding the publication of this paper. 


\section{Acknowledgments}

The authors are grateful to those who have helped during the writing of this paper. The research and publication of their article was funded by the National Natural Science Foundation of China (grant no. 51478289).

\section{References}

[1] M. A. Biot, "Theory of propagation of elastic waves in a fluidsaturated porous solid. II. Higher frequency range," The Journal of the Acoustical Society of America, vol. 28, no. 3, pp. 179-191, 1956.

[2] M. A. Biot, "Mechanics of deformation and acoustic propagation in porous media," Journal of Applied Physics, vol. 33, no. 4 , pp. $1482-1498,1962$.

[3] A. Philippacopoulos, "Lamb's problem for fluid-saturated, porous media," Bulletin of the Seismological Society of America, vol. 78, no. 2, pp. 908-923, 1988.

[4] J. T. Yazdi, S. Valliappan, and C. B. Zhao, "Analytical and numerical solutions for wave propagation in water-saturated porous layered half-space," Soil Dynamics and Earthquake Engineering, vol. 13, no. 4, pp. 249-257, 1994.

[5] B. Jin and H. Liu, "Dynamic response of a poroelastic half space to horizontal buried loading," International Journal of Solids and Structures, vol. 38, no. 44-45, pp. 8053-8064, 2001.

[6] A. H. D. Cheng, T. Badmus, and D. E. Beskos, "Integral equation for dynamic poroelasticity in frequency domain with BEM solution," Journal of Engineering Mechanics, vol. 117, no. 5, pp. 1136-1157, 1991.

[7] A. Mesgouez and G. Lefeuve-Mesgouez, "Transient solution for multilayered poroviscoelastic media obtained by an exact stiffness matrix formulation," International Journal for $\mathrm{Nu}$ merical and Analytical Methods in Geomechanics, vol. 33, no. 18, pp. 1911-1931, 2009.

[8] L. Wang and S. I. Rokhlin, "Stable reformulation of transfer matrix method for wave propagation in layered anisotropic media," Ultrasonics, vol. 39, no. 6, pp. 413-424, 2001.

[9] D. Pei, J. N. Louie, and S. K. Pullammanappallil, "Improvements on computation of phase velocities of Rayleigh waves based on the generalized R/T coefficient method," Bulletin of the Seismological Society of America, vol. 98, no. 1, pp. 280287, 2008.

[10] T. Liu and C. Zhao, "Dynamic analyses of multilayered poroelastic media using the generalized transfer matrix method," Soil Dynamics and Earthquake Engineering, vol. 48, pp. 15-24, 2013.

[11] K. Tuncay and M. Y. Corapcioglu, "Wave propagation in poroelastic media saturated by two fluids," Journal of Applied Mechanics, vol. 64, no. 2, pp. 313-320, 1997.

[12] B. Gatmiri and E. Jabbari, "Time-domain Green's functions for unsaturated soils. Part I: two-dimensional solution," International Journal of Solids and Structures, vol. 42, no. 23, pp. 5971-5990, 2005.

[13] W.-C. Lo, "Propagation and attenuation of Rayleigh waves in a semi-infinite unsaturated poroelastic medium," Advances in Water Resources, vol. 31, no. 10, pp. 1399-1410, 2008.

[14] M. Xu, Investigation on dynamic response of unsaturated soils and foundation, Ph.D. thesis, South China University Technology, Guangzhou, China, 2010, in Chinese.

[15] M. Zhang, X. Wang, G. Yang, and L. Xie, "Solution of dynamic Green's function for unsaturated soil under internal excitation," Soil Dynamics and Earthquake Engineering, vol. 64, pp. 63-84, 2014.
[16] R. Boer, W. Ehlers, and Z. Liu, "One-dimensional transient wave propagation in fluid-saturated incompressible porous media," Archive of Applied Mechanics, vol. 63, no. 1, pp. 59-72, 1993.

[17] M. Schanz and A.-D. Cheng, "Transient wave propagation in a one-dimensional poroelastic column," Acta Mechanica, vol. 145, no. 1-4, pp. 1-18, 2000.

[18] M. T. Van Genuchten, "A closed-form equation for predicting the hydraulic conductivity of unsaturated soils," Soil Science Society of America Journal, vol. 44, no. 5, pp. 892-898, 1980.

[19] F. Durbin, "Numerical inversion of Laplace transforms: an efficient improvement to Dubner and Abate's method," The Computer Journal, vol. 17, no. 4, pp. 371-376, 1974.

[20] B. Gatmiri and E. Jabbari, “Time-domain Green's functions for unsaturated soils. Part II: three-dimensional solution," International Journal of Solids and Structures, vol. 42, no. 23, pp. 5991-6002, 2005. 